\title{
Fisioterapia Nefrológica: Mejora De La Calidad De Vida Mediante Un Programa De Acondicionamiento Físico En Pacientes Con Enfermedad Renal Crónica En Una Unidad De Hemodiálisis En Mexico
}

\author{
Itzel Yeyetzin González Ovando, \\ Genaro Vega Malagón,
}

Licenciatura en Fisioterapia de la Facultad de Enfermería de la Universidad Autónoma de Querétaro

doi: 10.19044/esj.2017.v13n24p405 URL:http://dx.doi.org/10.19044/esj.2017.v13n24p405

\begin{abstract}
Objective: To analyze the improvement of the quality of life in the physical sphere through an effective physiotherapy program of physical conditioning in patients with Chronic Renal Disease in the Hemodialysis Unit in México. Methodology: Prospective longitudinal comparative study, pre and post treatment, in which the efficacy of a physiotherapeutic intervention and treatment and its impact on the quality of life during 20 weeks in a group of 25 patients with chronic renal disease undergoing hemodialysis were quantified. To evaluate the efficacy of the treatment, the study was divided into 5 stages and the KDQOL-SFTM questionnaire. Results: Results: In the physical sphere measured by the applied KDQOLSF TM instrument, the best results have been obtained, since statistically significant differences ( $p<0.05)$ were found between the variables of the decrease in difficulty in bending and bending $\mathrm{M} 2.28 \mathrm{DE}(0.842)$ versus $\mathrm{M}$ 2.64 DE (0.700) P 0.047, loads and transfers M 2.08 D.E (0.953) versus M 2.48 D.E (0.714) P 0.015, and decrease in difficulty in bathing and dressing (MV) M 2.56 D.E. (0.711) versus M 2.88 D.E (0.331) P 0.029, variables analyzed in the pre and post physiotherapeutic intervention, respectively. Conclusion: All patients showed a significant improvement in strengthresistance thanks to the physical-fitness program intradiálisis, increased the functional capacity and improved the physical sphere component of the quality of life of patients with Chronic Renal Disease.
\end{abstract}

Keywords: Nephrological Physiotherapy, Physical Conditioning, Quality of Life, Chronic Kidney Disease, Haemodialysis. 


\section{Resumen}

Objetivo: Analizar la mejora de la calidad de vida en la esfera física mediante un programa fisioterapéutico eficaz de acondicionamiento físico en pacientes con Enfermedad Renal Crónica en una Unidad de Hemodiálisis en México. Metodología: Estudio prospectivo comparativo longitudinal, experimental pre y postratamiento en el que se ha cuantificado la eficacia de una intervención y tratamiento fisioterapéutico y su impacto en la calidad de vida durante 20 semanas en un grupo de 25 pacientes con Enfermedad Renal Crónica sometidos a hemodiálisis. Para evaluar la eficacia del tratamiento el estudio se dividió en 5 etapas y se aplicó el cuestionario KDQOL-SFTM Resultados: En la esfera física medida por el instrumento KDQOLSFTM aplicado es donde se han conseguido los mejores resultados, ya que se han encontrado diferencias estadísticamente significativas $(\mathrm{p}<0,05)$ entre las variables de la disminución en la dificultad para agacharse e inclinarse $M$ 2.28 D.E (0.842) versus M 2.64 D.E. (0.700) P 0.047, cargas y traslados $\mathrm{M}$ 2.08 D.E (0.953) versus M 2.48 D.E (0.714) P 0.015, y en disminución en la dificultad para bañarse y vestirse (AVD) M 2.56 D.E. (0.711) versus M 2.88 D.E (0.331) P 0.029, variables analizadas en la pre y post intervención fisioterapéutica respectivamente. Conclusiones: Todos los pacientes mostraron una mejoría significativa en la fuerza-resistencia gracias al programa de acondicionamiento físico intradiálisis, aumentó la capacidad funcional y mejoró el componente de la esfera física de la calidad de vida de los pacientes con Enfermedad Renal Crónica.

Palabras-claves: Fisioterapia nefrológica, Acondicionamiento físico, Calidad de vida, Enfermedad Renal Crónica, Hemodiálisis.

\section{Introduction}

"Resulta alarmante que en el transcurso de 20 años la frecuencia de la Enfermedad Renal Crónica (ERC) a nivel Latinoamérica se ha incrementado cinco veces; quinientos de cien mil habitantes ya la padecen" Sabath, E. (2014) En México, existen evidencias de pocos estudios de fisioterapia renal; el paciente en hemodiálisis (HD) supone un importante gasto sanitario en su tratamiento sustitutivo fruto de la alta comorbilidad, lo cual puede limitar la inversión añadida en su tratamiento fisioterapéutico.

El ejercicio físico desempeña un papel importante en la mejora de la sensación de bienestar general del paciente. Más del 70\% de los pacientes en HD presentan síntomas articulares, y la prevalencia aumenta con el número de años. Los depósitos de ß-2-microglobulina producen dolor articular y dificultad de movimiento, así como síndrome del túnel carpiano y espondiloartropatía destructiva. 
Son múltiples los cristales intraarticulares que pueden producir inflamación articular y debilidad muscular, Daugirdas, B., (2003). Tras 30 años de investigación sobre los efectos del ejercicio en pacientes en HD parece que éste es seguro, y que la modalidad durante la HD es la que mayor seguimiento consigue por parte del paciente a pesar de que la implantación de programas de ejercicio en unidades de HD no está generalizada en la mayoría de países, Segura (2010).

Según datos de la National Kidney Foundation (2007) "la diabetes es la principal causa de la ERC y constituye el $45 \%$ de los casos, en todo el mundo, al menos 171 millones de personas la padecen”. En México, se cuenta con 10.6 millones de diabéticos, con una prevalencia de 8 de cada 100 personas mayores de 20 años, que incrementa con la edad, calculándose que posterior a los 50 años, son 20 de cada 100. Asimismo, cifras del Instituto Nacional de Geográfica e Información (INEGI, 2011), refieren que esta patología es la segunda causa de mortalidad según datos recabados del Boletín epidemiológico de la Secretaría de Salud (2015).

Existen datos de que pacientes sometidos a HD tienen bajos niveles de actividad física y que los datos de hospitalización y de supervivencia son directamente proporcionales a la actividad física; hasta el punto de que un paciente de 30 años sometido a HD tiene menos actividad física diaria que un individuo sano y sedentario de 70 años de edad, Hervás (2011).

Los pacientes en HD con alteración de la función cardiovascular, es responsable del $50 \%$ de muertes en pacientes con ERC. Las alteraciones musculares que padecen estos pacientes son el principal factor limitante de la capacidad funcional, siendo frecuentes la debilidad muscular, fatiga, mioclonías y los espasmos musculares siendo éstas los factores limitantes más importantes de su capacidad funcional, Oliveros et al (2011).

La ERC es consecuencia de una nefropatía glomerular, nefroangiosclerosis o de una nefropatía intersticial. El cuadro asintomático asocia perturbaciones electrolíticas, acidosis metabólica, esclerosis vascular y anemia, Xhardez, Yves (2002). Las alteraciones a nivel bioquímico provocan síntomas que sufren diariamente y que limita drásticamente su capacidad para trabajar o realizar actividades de ocio.

El estilo de vida sedentario es una de las principales causas de la baja capacidad física y un factor de riesgo independiente de muerte en pacientes con ERC sometidos a HD, Carrero et al (2008). Además hay una declinación progresiva de la capacidad funcional después del inicio de programas de HD crónica según demostró Kurella et al (2010).

El ejercicio aeróbico y de resistencia muscular durante la diálisis aumentan el flujo sanguíneo a nivel muscular con aumento del área de superficie capilar, lo que dinamiza el flujo de la urea y toxinas desde los tejidos hacia el compartimiento vascular, lo que pudiera mejorar la eficacia 
de la HD según Parsons, Toffelmire, King-Van-Vlack (2006). Además el ejercicio regular moderado puede modular la respuesta inflamatoria, lo que sería beneficioso en ERC demostró Beavers, Brinkley, Nicklas (2010).

El fisioterapeuta se enfrenta a patologías como: sideración e hipotonía muscular generalizada, osteodistrofia renal, polineuritis urémica, miopatía urémica, necrosis aséptica de la cabeza femoral, complicaciones cardíacas, musculares, respiratorias, psicológicas, periartritis escapulohumeral, tenosinovitis de los flexores, espondiloartropatía destructiva, seudotumor del periodontoides, depósitos amiloides extradurales, quistes óseos, fracturas patológicas, artritis inducida por cristales, artritis reumatoide, gota, condrocalcinosis, osteonecrosis, bursitis del olecranon, dermopatía fibrosante, lupus eritematoso sistémico, síndrome de las piernas inquietas, mononeuropatías y las ya mencionadas por neuropatía urémica, nefropatía diabética e hipertensión. El ejercicio tiene un impacto favorable sobre los valores de colesterol y triglicéridos, así como el incremento de la sensibilidad a la insulina; aumenta la fibrinólisis, eleva la captación de oxígeno tanto en el corazón como en los tejidos, incrementa la circulación periférica, aumenta el umbral de fibrilación ventricular, y disminuye la frecuencia cardíaca al aumentar el tono vagal y la agregación plaquetaria; todos estos cambios apuntan a un menor riesgo aterotrombótico y una menor probabilidad de muerte por causa cardiovascular, Serra, Díaz, Sande (2005).

Existe evidencia que un programa adaptado de ejercicio físico de baja intensidad en el adulto mayor mejoró la fuerza muscular, la capacidad funcional, la sintomatología depresiva y la calidad de vida, Esteve et al. (2015). Este incremento de fuerza podría corresponder a cambios morfológicos y funcionales de las fibras musculares que conllevarían una mayor activación y reclutamiento de los grupos musculares implicados y, en consecuencia, una mayor fuerza de ellos, Johansen et al (2003).

Coincidimos con Kutner et al, Fitts et al y Koudi et al en que los planes fisioterapéuticos en el paciente renal en cualquiera de sus fases mejoran su fuerza muscular, su resistencia dinámica, su independencia en actividades de la vida diaria, aumento de movilidad, mejora de funcionalidad y de percepción en la mejora de la calidad de vida relacionada a la salud tal como lo demostró Peña, García, Zagalaz, Jimeno, Expósito (2009). Krause realizó en 2003 una sencilla encuesta a 47 nefrólogos y sus conclusiones señalan que aceptan que la actividad física es importante para producir beneficios de salud en los pacientes y disminuir factores de riesgo. La implementación de este tipo de programas de ejercicio en la unidad de HD debe ser considerada como una oportunidad única para mejorar la salud de los pacientes, Segura (2007). 
El objetivo principal de este estudio fue analizar la mejora de la calidad de vida en la esfera física mediante un programa fisioterapéutico de acondicionamiento en pacientes con Enfermedad Renal Crónica en la Unidad de Hemodiálisis de Servicios de Salud del Estado de Querétaro (SESEQ) durante el periodo comprendido de agosto a diciembre del 2015.

\section{Metodología}

Se realizó un estudio cuasi experimental, prospectivo, comparativo, y longitudinal, con el objetivo de evaluar la eficacia de una intervención y tratamiento fisioterapéutico de fuerza-resistencia y su impacto en la calidad de vida durante 20 semanas en un grupo de 25 pacientes con Enfermedad Renal sometidos a hemodiálisis que asisten a la Unidad de Hemodiálisis en México.

Para evaluar la eficacia del tratamiento el estudio se dividió en 5 etapas: Etapa preliminar donde se realizó una valoración fisioterapéutica inicial empleando cada sujeto de la muestra como su propio control y la aplicación del cuestionario KDQOL-SF ${ }^{\mathbf{T M}}$. Etapa pre intervención fisioterapéutica donde se aplicaron y registraron los resultados de las siguientes pruebas de condición física: Test de los 6MWT, Balance muscular, Prueba del STS-10, Contracción isométrica de cuádriceps y Extensión de rodilla. Etapa de intervención de ejercicio terapéutico con un programa personalizado durante la sesión y en casa. Etapa de valoración post intervención fisioterapéutica donde se repitieron las pruebas de condición física mencionadas anteriormente y nuevamente la aplicación de cuestionario KDQOL-SF ${ }^{\mathrm{TM}}$. La quinta etapa de interpretación de datos.

Se solicitó a los miembros del equipo sanitario un listado con los pacientes que podían ser susceptibles de participar en el estudio. Los criterios de elegibilidad fueron los siguientes: edad comprendida entre 19 y 87 años, al menos 6 meses en tratamiento en HD, encontrarse estable clínicamente con una autorización previa del médico nefrólogo, que asistiera a terapia de HD 2 veces por semana y por último una autorización del paciente por escrito junto con un consentimiento informado.

Fueron considerados como criterios de exclusión los siguientes: ser portadores de accesos vasculares en miembros inferiores, tener algún miembro inferior amputado y por último, tener diagnosticado algún problema cardiovascular previo que pudiera empeorar con el ejercicio. Una vez aplicados los criterios de inclusión y exclusión, el número de participantes se redujo de 50 a 25 participantes

La distribución de pacientes en la Unidad de HD es en 3 turnos de enfermos. Se eligieron a pacientes de los 3 turnos. Se inició y terminó el estudio con 25 sujetos, los cuales finalizaron el programa en tiempo y forma. La intervención fisioterapéutica se realizó durante las 2 primeras horas de 
HD, la sesión duraba al menos 50 minutos incluyendo calentamiento, flexibilización, acondicionamiento, vuelta a la calma y flexibilización final.

Las rutinas incluían gimnasia de extensión y de erección vertebral para control motor, fortalecimiento de la cincha abdominal, movilizaciones, estiramientos y fortalecimiento muscular (ejercicios isométricos) de los miembros, ejercicios respiratorios, ejercicios isotónicos con $3 \mathrm{~kg}$ de peso como máximo, ejercicios de resistencia progresiva con ligas y ejercicios de relajación/reducción del estrés y de retroalimentación (biofeedback).Los datos fueron analizados mediante el programa de análisis estadístico SPSS v.23. El cuestionario de calidad de vida se analizó con el cuestionario KDQOLSFTM Kidney Disease and Quality of Life Version 1.3 Short Form; Duarte, Miayzaki, Ciconelli, Sesso (2003), traducida del inglés al español. Para cada variable se han hallado los valores descriptivos de tendencia central y de dispersión más habituales (media, desviación estándar) que se presentan en sus correspondientes tablas. Se ha empleado el límite de $\mathrm{p}<$ 0,05 para considerar un resultado como significativo.

\section{Resultados}

La edad más fue en el grupo de 19 a 30 años, seguida del grupo de 31 a 50 años, en relación al sexo el comportamiento de la población fue similar.

VER CUADRO N $^{\circ}$ :

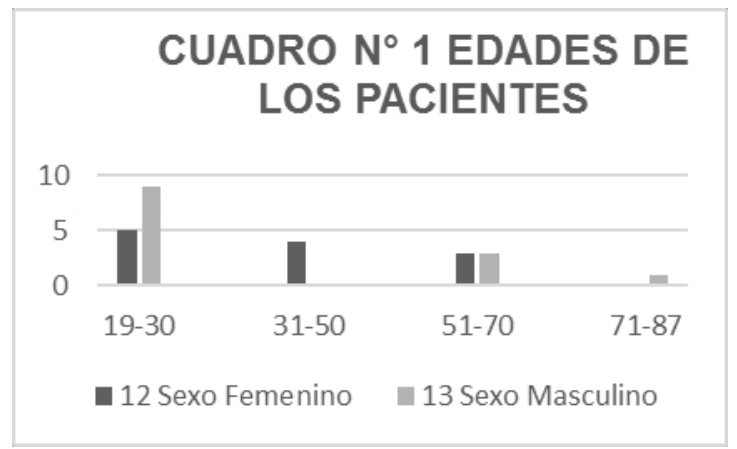

Los resultados de calidad de vida en el enfermo renal, una vez analizado el cuestionario según el programa KDQOLSFTM Version 1.3 Short Form Scoring Program, se exponen en el CUADRO № 2.

CUADRO N$^{\circ}$ 2.- Calidad de vida en esfera física

\begin{tabular}{cccccc}
\multicolumn{2}{c}{ Pre tratamiento } & \multicolumn{2}{c}{ Post tratamiento } & VALOR & IC 95\% \\
Media & (D.E.) & Media & (D.E.) & P* &
\end{tabular}




\begin{tabular}{|c|c|c|c|c|c|c|}
\hline $\begin{array}{l}\text { agacharse e } \\
\text { inclinarse }\end{array}$ & & & & & & \\
\hline $\begin{array}{l}\text { Cargas y } \\
\text { traslados }\end{array}$ & 2.08 & $(0.953)$ & 2.48 & $(0.714)$ & 0.015 & $-0.715--0.084$ \\
\hline $\begin{array}{c}\text { Dificultad para } \\
\text { bañarse y } \\
\text { vestirse }\end{array}$ & 2.56 & $(0.711)$ & 2.88 & $(0.331)$ & 0.029 & $-0.604--0.035$ \\
\hline $\begin{array}{c}\text { Actividad física } \\
\text { moderada }\end{array}$ & 2.16 & $(0.898)$ & 2.48 & $(0.770)$ & 0.103 & $-0.710-0.070$ \\
\hline $\begin{array}{c}\text { Dificultad para } \\
\text { caminar más de } \\
1 \mathrm{~km}\end{array}$ & 2.08 & $(0.909)$ & 2.44 & $(0.711)$ & 0.095 & $-0.787-0.067$ \\
\hline
\end{tabular}

P*: < de 0.05 Estadísticamente significativo (T de Student)

En la esfera física medida por el instrumento KDQOLSFTM aplicado es donde se han conseguido los mejores resultados, ya que se han encontrado diferencias estadísticamente significativas $(\mathrm{p}<0,05)$ entre las variables de la disminución en la dificultad para agacharse e inclinarse M 2.28 D.E (0.842) versus M 2.64 D.E. (0.700) P 0.047, cargas y traslados M 2.08 D.E (0.953) versus M 2.48 D.E (0.714) P 0.015, y en disminución en la dificultad para bañarse y vestirse (AVD) M $2.56 \quad$ D.E. (0.711) versus $\quad M \quad 2.88$ D.E (0.331) P 0.029 , variables analizadas en la pre y post intervención fisioterapéutica respectivamente, todos los sujetos experimentaron una mejoría en mayor o menor medida.

\section{Discusión}

Se han obtenido resultados muy positivos en la esfera física de calidad de vida del paciente renal, la intervención fisioterapéutica personalizada ha impactado en la fuerza y la capacidad funcional de los pacientes para la realización de las Actividades de la Vida Diaria (AVD) lo que sugiere que el programa de acondicionamiento físico durante la HD produjo una mejora en las cadenas musculares y en el aparato locomotor en general.

Este hallazgo coincide con las observaciones de estudios previos, que demostraron mejoras significativas en la eficacia de un programa de ejercicio intradiálisis tanto con la aplicación de un programa fisioterapéutico de fuerza-resistencia muscular como la aplicación de electro estimulación neuromuscular durante las sesiones. El estudio mencionado anteriormente incluyó únicamente a 10 pacientes que se sometieron al programa fisioterapéutico intradiálisis durante 5 semanas en el Complejo Hospitalario de Jaén, en la Unidad de Crónicos del Servicio de Nefrología, España, Contreras et al. (2011) frente a nuestro estudio de 25 pacientes con 20 semanas de entrenamiento en México. 
Además, la mejora de la calidad de vida se ha visto en otros estudios que han demostrado su relación con los efectos del ejercicio terapéutico en el paciente renal como el de Segura, Rodilla, Lisón (2008) que han aplicado programas exclusivos de fuerza en sujetos en diálisis. Su estudio incluyó a 16 pacientes con una media edad de 54 años a los cuales dividió en dos grupos: el de intervención con 8 pacientes y el de control, también con 8 (el grupo control fue formado por aquellos pacientes que se negaron a realizar el programa de entrenamiento). Aunque los resultados fueron similares a los del presente estudio, hay que destacar que a pesar de que la media de edad del estudio de Segura et al era de 54,9 frente a los 38,12 de media de nuestro estudio, y de que su programa de entrenamiento fue de 6 meses frente al nuestro de 5 meses, una diferencia notable es que nuestro estudio fue capaz de arrojar resultados más específicos de cada variable de la esfera física, pues la literatura de Segura et al no desglosa las variables evaluadas como lo es cargas y traslados, mejora en la dificultad de inclinación en el cambio de posturas básicas y la mejora de la dificultad para una AVD básica como es bañarse y vestirse; entre otras, variables importantes porque denotan una tendencia a la independencia del paciente nefrópata.

En cuanto a calidad de vida nuestro estudio ha obtenido resultados significativos en la esfera física, está claro que existe una asociación entre mayor facilidad para la realización de las AVD de forma independiente, y una mayor calidad de vida en la esfera física y mental - esta última no ha sido objeto de análisis en el presente estudio, Hays et al (1995). A pesar de esto, nos sorprende que en nuestro estudio no haya habido diferencias significativas como esperábamos en la variable de actividad física moderada la cual incluye caminar varias cuadras con una cadencia veloz, subir con mayor velocidad escaleras, bailar y realizar actividad laboral sin dificultad. Así como la variable de la dificultad al caminar más de un kilómetro; aunque este hecho se puede deber a que los pacientes se encontraban en un sedentarismo extremo cuando iniciamos la intervención, la edad y la elevada comorbilidad del paciente.

Sería interesante ampliar el presente estudio a una segunda etapa con los resultados de las pruebas físicas pre y post intervención fisioterapéutica incluyendo el efecto de los fármacos en la fisiología muscular del paciente. Este estudio pretende abrir nuevas posibles líneas de investigación ante la carencia de especialistas en el área nefrológica que representa un grave problema de salud pública no solamente del sector médico; los fisioterapeutas especializados en esta población sensible son prácticamente nulos, se debe definir el rol del fisioterapeuta en el área nefrológica para promover de manera urgente los programas de ejercicios especialmente diseñados para pacientes renales en los centros de diálisis. 
Este estudio pretendía analizar la mejora de la calidad de vida mediante un programa de acondicionamiento físico en pacientes con Enfermedad Renal Crónica en la Unidad de Hemodiálisis y determinar la eficacia de la intervención fisioterapéutica y su impacto en la esfera física a través de un programa de ejercicios de fuerza-resistencia durante la sesión, nuestros resultados han confirmado este objetivo principal cumpliéndose que un adecuado y personalizado programa de acondicionamiento físico permite el fortalecimiento muscular que se obtiene gracias a la realización del ejercicio el cual mejora la capacidad funcional y la realización de las AVD tales como andar, caminar, pasar de sedente a bipedestación, realizar cargas, traslados, bañarse y vestirse.

Conclusión, un programa de acondicionamiento físico intradiálisis aumentó la capacidad funcional y mejoró el componente físico de la calidad de vida de los pacientes, por lo que se justificaría la incorporación a los tratamientos habituales de diálisis una pauta de ejercicios totalmente personalizada pues el presente estudio aportó evidencia de que el entrenamiento de fuerza posee un efecto positivo sobre la calidad de vida relacionada con la salud. Se recomienda que el ejercicio durante la HD se realice en las primeras 2 horas de tratamiento; puede adaptarse a cualquier paciente y en todos los casos se pueden obtener beneficios.

\section{References:}

1. Beavers KM, Brinkley TE, Nicklas BJ. (2010) Effect of exercise training on chronic inflammation. Clin Chim Acta; 411: 785-93.

2. Boletín epidemiológico de la Secretaría de Salud. Número 13 Volumen 32 | Semana 13 (29 de marzo al 4 de abril del 2015). "Transición epidemiológica" Recuperado de:http://www.epidemiologia.salud.gob.mx/doctos/boletin/2015/sem 13.pdf

3. Carrero, J., Chmielewski, M., Axelsson, J., Snaedal S, Heimbürger O, Bárány (2008) P. Muscle atrophy, inflammation and clinical outcome in incident and prevalent dialysis patients. Clin Nutr 27: 557-64.

4. Contreras, G., et al. (2011) Eficacia de un programa de entrenamiento intradiálisis de fuerza-resistencia en combinación con electroestimulación neuromuscular: mejora en la capacidad funcional, fuerza, y calidad de vida. Rev Soc Esp Enferm Nefrol; 14 (2): 112/119

5. Daugirdas, B., (2003), Manual de Diálisis, 2ª edición. Barcelona, España. Editorial Masson. 
6. Duarte SP, Miayzaki MCOS, Ciconelli RM, Sesso R. (2003) Tradução e adaptação cultural do instrumento de avaliação de qualidade de vida para pacientes renais crónicos (KDQOL-SF ${ }^{\mathrm{TM}}$ ). Rev Assoc Med Bras (São Paulo);49(4):375-81.Rescatado de: http://www.scielo.br/pdf/ramb/v49n4/18335.pdf

7. Esteve, S., et al. (2015). Beneficios del ejercicio físico de baja intensidad durante la sesión de hemodiálisis en el paciente anciano. Nefrología Madr. Vol. $35 \mathrm{~N}^{\mathrm{o}}$ 4. Recuperado de: http://dx.doi.org/10.1016/j.nefro.2015.03.006

8. Hays RD, Kallich JD, Mapes DL, Coons SJ, Armin N, y Carter WB. Kidney Disease Quality of Life Short Form (KDQOL-SFTM), Version 1.3: A manual for Use and Scoring. 1995; Santa Mónica, CA: RAND, P-7994.

9. Hervás, J. (2011). XXXIII Annual Congress of the Spanish Society of Dialysis and Transplantation. Granada. Vol. 32. Núm. 01. Enero 2011 - Marzo 2011

10. Johansen KL, Shubert T, Doyle J, Soher B, Sakkas GK, Kent-Braun (2003). Muscle atrophy in patients receiving hemodialysis: Effects on muscle strenght, muscle quality, and physical function. Kidney int. 63(1):291-7

11. Kurella-Tamura M, Covinsky KE, Chertow GM, Yaffe K, Landefeld CS, McCulloch CE. (2010) Functional status of elderly adults before and after initiation of dialysis. N Engl J Med 361: 1539-47.

12. National Kidney Foundation, Inc. (2007). La diabetes y la Insuficiencia Renal Crónica (Falla Crónica del Riñón, etapa V). Estados Unidos. Recuperado de: www.kidney.org

13. Oliveros R, María Soledad, Avendaño, Marcelo, Bunout, Daniel, Hirsch, Sandra, De La Maza, María Pía, Pedreros, Cristian, \& Müller, Hans. (2011). Estudio piloto sobre entrenamiento físico durante hemodiálisis. Revista médica de Chile, 139(8), 1046-1053.

14. Parsons TL, Toffelmire EB, King-Van-Vlack CE. (2006) Exercise training during hemodialysis improves dialysis efficacy and physical performance. Arch Phys Med Rehabil 87: 680-7.

15. Peña, P.; García, J.; Zagalaz, M.; Jimeno, R.; Expósito, A. (2009) El ejercicio físico en pacientes en insuficiencia renal crónica terminal y programa de hemodiálisis. Universidad de Jaen, España. Vol. 30. Núm. 4. Dial Traspl 30:127-32 - DOI: 10.1016/S18862845(09)72696-7

16. Sabath, E. (Octubre 15 de 2014) Periódico Diario de Querétaro, Derechos Reservados "Dr. Ernesto Sabath Silva Director de la Clínica de Hemodiálisis". Querétaro, México. Organización Editorial 
Mexicana S.A. de C.V. Recuperado de: http://www.oem.com.mx/diariodequeretaro/notas/n3571672.htm

17. Segura E, Rodilla-Alama V, Lisón JF. (2008) Fisioterapia durante la hemodiálisis: resultados de un programa de fuerza-resistencia. Nefrología; 28 (1): 67-72.

18. Segura, E. (2007) Programa de ejercicio para pacientes con insuficiencia renal crónica en hemodiálisis. Estudio piloto. Rev Soc Esp Enferm Nefrol; 10 (3): 244/246

19. Segura, E. Ejercicio en pacientes en hemodiálisis: revisión sistemática de la literatura. Depto de Fisioterapia. Universidad CEU, Moncada, Valencia. Nefrología 2010;30(2):236-46

20. Serra,G.; Díaz, J.; Sande, C. (2005) Fisioterapia en neurología, sistema respiratorio y aparato cardiovascular. Madrid, España. Editorial Masson. Elsevier.

21. Xhardez, Yves. (2002) Vademecum de kinesioterapia y de reeducación funcional: técnicas, patología e indicaciones de tratamiento $4^{\mathrm{a}}$ edición, $3^{\circ}$ reimpresión. Buenos Aires, Editorial El Ateneo. 